\title{
SOLAR-TYPE POST-T TAURI STARS IN THE NEAREST OB SUBGROUPS
}

\author{
Eric E. Mamajek \\ Steward Observatory, The University of Arizona, \\ 933 N. Cherry Ave., Tucson AZ 85721 \\ eem@as.arizona.edu
}

\begin{abstract}
I discuss results from the recent spectroscopic survey for solar-type preMS stars in the Lower Centaurus-Crux (LCC) and Upper CentaurusLupus (UCL) OB subgroups by Mamajek, Meyer, \& Liebert (2002, AJ, $124,1670)$. LCC and UCL are subgroups of the Sco-Cen OB association, and the two nearest OB subgroups to the Sun. In the entire survey of 110 pre-main sequence stars, there exists only one Classical $\mathrm{T}$ Tauri star (PDS 66 ), implying that only $\sim 1 \%$ of $\sim 1 \mathrm{M}_{\odot}$ stars are still accreting at age $13 \pm 7(1 \sigma)$ Myr. Accounting for observational errors, the HRD placement of the pre-MS stars is consistent with the bulk of star-formation taking place within 5-10 Myr. In this contribution, I estimate conservative upper limits to the intrinsic velocity dispersions of the post-T Tauri stars in the LCC and UCL subgroups $\left(<1.6 \mathrm{~km} \mathrm{~s}^{-1}\right.$ and $<2.2 \mathrm{~km} \mathrm{~s}^{-1}$, respectively; $95 \% \mathrm{CL}$ ) using Monte Carlo simulations of Tycho- 2 proper motions for candidate subgroup members. I also demonstrate that a new $\mathrm{OB}$ subgroup recently proposed to exist in Chamaeleon probably does not.
\end{abstract}

\section{Introduction}

In understanding the development of our own solar system and the formation of stars and planets in general, we would like to know: How long does star-formation persist in molecular clouds? How long do stars accrete from circumstellar disks? What controls the rotational evolution of pre-main sequence stars? What are the characteristics and frequency of dusty debris disks around solar-type stars? General questions regarding star and planet formation can be addressed by identifying and investigating large samples of pre-main sequence stars in the nearest OB associations. Understanding the evolution of low-mass stars intermediate in age between embedded T Tauri stars (TTSs) and zero-age 
main sequence (ZAMS) stars has been historically impeded by the lack of appropriate stellar samples. T Tauri stars ( $\leq$ few Myr-old) are found in great numbers in and near molecular clouds, while well-characterized ZAMS stars ( 30-100 Myr) are abundant in nearby open clusters. Finding the elusive, intermediate-age, pre-MS "post-T Tauri" stars has recently become possible due to the availability of the ROSAT All-Sky Survey and Hipparcos/Tycho databases (Jensen 2001).

In this contribution, I summarize the findings of a recent spectroscopic survey of post-T Tauri stars in the Lower Centaurus-Crux (LCC) and Upper Centaurus-Lupus (UCL) subgroups (Mamajek, Meyer, \& Liebert, 2002; hereafter MML02). These are the two oldest subgroups of the ScoCen OB association, and the two nearest OB subgroups to the Sun. I also calculate an upper limit to the velocity dispersion of low-mass members of LCC and UCL, and critically examine the evidence for a new OB subgroup in Chamaeleon.

\section{Post-T Tauri Stars in LCC and UCL}

MML02 conducted a spectroscopic survey of a proper motion- and Xray-selected sample of stars in the LCC and UCL regions of the Sco-Cen OB association. For the survey, MML02 used the Astrographic CatalogTycho (ACT) and Tycho Reference Catalog (TRC) astrometric catalogs and the ROSAT All-Sky Survey Bright Source Catalog (RASS-BSC) of $\mathrm{X}$-ray sources. The proper motion candidates were selected by Hoogerwerf (2000) and MML02 as being stars whose both ACT and TRC proper motions were consistent with LCC or UCL membership, and whose B-V colors and $\mathrm{V}$ magnitudes were between $3 \mathrm{mag}$ above and 1 mag below the Schmidt-Kaler (1982) ZAMS at the mean distance for each OB subgroup. To narrow our selection of young stellar candidates, we observed only those stars that had X-ray sources within 40" radius in the RASSBSC. We added to our target list the G and K-type Hipparcos stars selected by de Zeeuw et al (1999) as probable LCC and UCL members. Blue and red medium resolution spectra were taken with the Dual-Beam Spectrograph on the Siding Springs 2.3-m telescope in April 2000. From the proper motion- and X-ray-selected sample, we identified the preMS stars spectroscopically through the following criteria: late spectral types (FGK), Li-rich (Li I $\lambda 6707$ line), subgiant surface gravities (using a band-ratio measurement of the Sr II $\lambda 4077$ and Fe I $\lambda 4071$ absorption lines), and HRD positions above the main sequence. The success rate for detecting Li-rich subgiants (i.e. probable pre-MS stars) among the proper motion- and X-ray selected sample was $93 \%$, compared to $73 \%$ 
for the de Zeeuw et al. (1999) kinematic sample. Only one star in the sample (MML $34=$ PDS 66) had strong $\mathrm{H} \alpha$ emission and a statisticallysignificant K-band excess consistent with being a Classical T Tauri star. The MML02 survey demonstrated the following:

- The mean pre-MS isochronal ages of LCC and UCL are nearly identical, and agree well with new estimates of the turn-off ages ( 15-23 Myr, depending on choice of evolutionary tracks).

- Only $\sim 1 \%$ of solar-type stars in our sample are Classical T Tauri stars. The mean age for the sample, biased toward younger ages for the lower mass stars, is 13 Myr using the D'Antona \& Mazzitelli (1997) tracks. The incidence of accretion disks is consistent with the idea that accretion terminates in solar-type stars within a $\sim 10$ Myr timescale.

- The band ratio Sr II $\lambda 4077 / \mathrm{Fe}$ I $\lambda 4071$ is very useful for segregating Li-rich stars into pre-MS and ZAMS stars. This band-ratio defines clear loci for dwarfs and subgiants among spectral standards.

- $95 \%$ of the low-mass star-formation in each OB subgroup must have taken place within a 8-12 Myr span.

\section{The Velocity Dispersion of the LCC and UCL Post-T Tauri Stars}

With a new, high-quality astrometric catalog now available (Tycho2 ), one can address the question: is the internal velocity dispersion of the post- $T$ Tauri members the same as that for the high mass members (<1-1.5 $\mathrm{km} \mathrm{s}^{-1}$; de Bruijne 1999a)? Is it measurable with existing data? Our group has taken echelle spectra of all of the MML02 pre-MS stars, with one goal being to measure the velocity dispersion and possible expansion of the OB subgroups. One can, however, calculate an upper limit to the velocity dispersion with the Tycho-2 astrometry alone.

Pre-main sequence members of the OB subgroups can be efficiently selected by their strong X-ray emission and convergent proper motions (MML02). To identify low-mass member candidates, I construct a crossreferenced catalog of all Tycho-2 stars with ROSAT All-sky Survey BSC and FSC X-ray sources within 40" radius (hereafter RASS-TYC2), and analyze the distribution of their proper motions. Within the LCC and UCL regions (defined by de Zeeuw et al. 1999), I find 271 RASS-TYC2 stars with $(\mathrm{B}-\mathrm{V})_{J}>0.60$ (G-type or later) in LCC and 328 in UCL. For simplicity, I do not apply a magnitude restriction other than the 
Tycho-2 magnitude limit - the vast majority are consistent with being pre-MS or ZAMS at $d=100-200$ pc.

To search for subgroup members, I plot the proper motions for the RASS-TYC2 stars in $\left(\mu_{v}, \mu_{\tau}\right)$ space instead of $\left(\mu_{\alpha}, \mu_{\delta}\right)$. The proper motion components represent the motion toward the subgroup convergent point $\left(\mu_{v}\right)$ and perpendicular to the great circle between the star and the convergent point $\left(\mu_{\tau}\right)$ (Smart 1968). The expectation value of $\mu_{\tau}$ for an ensemble of bona fide cluster members is zero, and $\mu_{v}$ scales with distance and angular separation from the convergent point. I adopt the space motion for the LCC and UCL subgroups from de Bruijne (1999a) (the $g_{\text {lim }}=9$ solutions in their Table 5 , where the space motion vector can be converted to a convergent point solution using eqn. 10 of de Bruijne (1999b)). Low-mass members of the OB subgroups stand out clearly in $\left(\mu_{v}, \mu_{\tau}\right)$ space (Fig. 1). The mean subgroup proper motion values (de Bruijne 1999a; Table 4) are shown as dashed vertical lines, where $\bar{\mu}$ for members is approximately equal to $\bar{\mu}_{v}$. I define boxes around the loci in Fig. 1 to select probable association members for statistical study (70 stars in LCC, 105 in UCL).

In order to estimate the observed dispersion in $\mu_{\tau}$ in a way that is insensitive to the boundaries of the subjectively drawn selection box (Fig. 1), and the presense of outliers, I use probability plots (Fig. 2). I follow the analysis method outlined in $\S 3$ of Lutz \& Upgren (1980). In a probability (or "probit") plot, the abscissa is the expected deviation from the mean predicted for the $i$ th sorted data point in units of the standard deviation, and the ordinate is the data value in question $\left(\mu_{\tau}\right)$. The slope of the probability plot distribution yields the standard deviation, and the $y$-intercept is the median. To fit a line to the probability plots in Fig. 2, I use the Numerical Recipes least-squares routine fit, and trim $10 \%$ from both sides of the distribution to mitigate against the effects of outliers. The probability plots yield observed standard deviations of $\sigma\left(\mu_{\tau}\right)=2.9 \pm 0.5 \mathrm{mas} \mathrm{yr}^{-1}$ (LCC) and $3.5 \pm 0.5 \mathrm{mas} \mathrm{yr}^{-1}$ (UCL). The mean values of $\mu_{\tau}$ are close to zero $\left(0.7 \pm 0.3\right.$ mas yr $^{-1}$ for LCC, $0.3 \pm 0.3$ mas yr$^{-1}$ for UCL), consistent with the expectation that most of the RASS-TYC2 stars in the boxes in Fig. 1 are subgroup members.

An upper limit to the velocity dispersions of the low-mass subgroup memberships can be estimated as follows. The observational errors in $\mu_{\tau}$

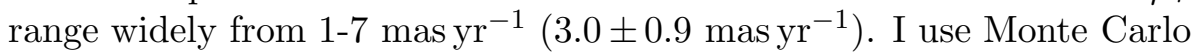
simulations to estimate the observed scatter expected in $\mu_{\tau}$ accounting 

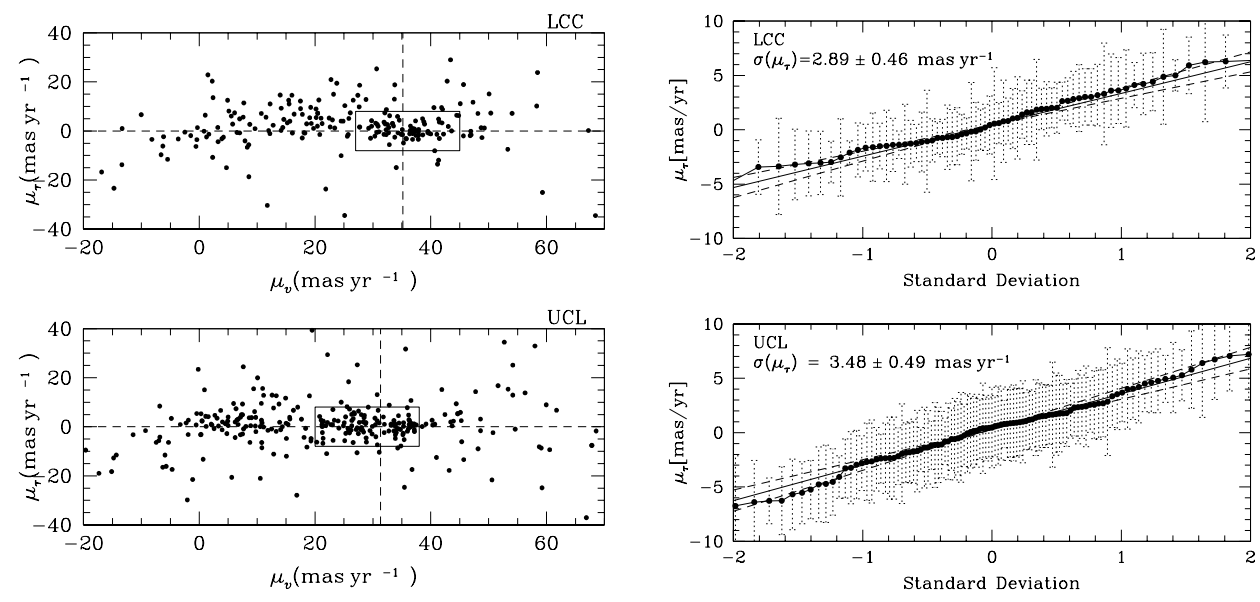

Figure 1. Proper motions of RASSTYC2 stars with $(\mathrm{B}-\mathrm{V})_{J}>0.60$, lying within the sky regions of the LCC and UCL OB subgroups defined by de Zeeuw et al. (1999). The mean $\bar{\mu}_{v}$ values for the OB subgroups are shown by vertical dashed line (from de Bruijne 2000). Association members should have a mean value of $\bar{\mu}_{\tau}=0$, with small scatter $\sigma\left(\mu_{\tau}\right)$.

Figure 2. Probability plots for $\mu_{\tau}$ for stars in the boxes defined in Fig. 1. A Gaussian profile will produce a straight line, where the slope gives the standard deviation. The observed distributions are consistent with the Tycho-2 proper motion errors combined with intrinsic velocity dispersions of $0.6_{-0.6}^{+0.5} \mathrm{~km} \mathrm{~s}^{-1}$ (LCC) and $1.0_{-1.0}^{+0.6} \mathrm{~km} \mathrm{~s}^{-1}$ (UCL).

for both the Tycho-2 proper motion errors and the intrinsic velocity dispersion of the association. The intrinsic velocity dispersion $\sigma_{\text {int }}^{\star}$ (in

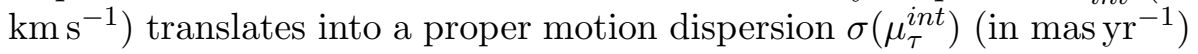
as a function of mean subgroup parallax $\pi$ (adapted from de Bruijne (1999) eqn. 20):

$$
\sigma\left(\mu_{\tau}^{i n t}\right)=\pi \sigma_{\text {int }}^{\star} / A
$$

where $\mathrm{A}=4.74 \mathrm{~km} \mathrm{~s}^{-1} \mathrm{yr}^{-1}$. For the simulations, I model velocity dispersions ranging from $\sigma_{\text {int }}^{\star}=0-3 \mathrm{~km} \mathrm{~s}^{-1}$ in $0.5 \mathrm{~km} \mathrm{~s}^{-1}$ steps. I adopt the mean distances to LCC and UCL from de Zeeuw et al. (1999). I generate $10^{4}$ Gaussian deviates for each star with zero mean and a standard deviation equal to the square root of the observed value of $\sigma\left(\mu_{\tau}\right)$ and the model $\sigma\left(\mu_{\tau}^{i n t}\right)$ values added in quadrature. Statistical testing showed that clipping the Monte Carlo data at the box boundaries in Fig. $1\left(\left|\mu_{\tau}\right|<8\right.$ mas yr $\left.^{-1}\right)$ had negligible effect on the probability plot determinations of $\sigma\left(\mu_{\tau}\right)$, so all Monte Carlo values were retained. 
A comparison between the $\sigma\left(\mu_{\tau}\right)$ values for the Monte Carlo simulations and the observations is shown in Table 1. It appears that the internal velocity dispersions of the subgroups are indeed detectable. The observed $\sigma\left(\mu_{\tau}\right)$ values for LCC and UCL are consistent with internal velocity dispersions of $\sigma_{\text {int }}^{\star}=0.6_{-0.6}^{+0.5} \mathrm{~km} \mathrm{~s}^{-1}$ and $1.0_{-1.0}^{+0.6} \mathrm{~km} \mathrm{~s}^{-1}$, respectively. The $95 \%$ confidence level upper limits to the velocity dispersions are $<1.6 \mathrm{~km} \mathrm{~s}^{-1}$ (LCC) and $<2.2 \mathrm{~km} \mathrm{~s}^{-1}$ (UCL). We can rule out velocity dispersions of $3 \mathrm{~km} \mathrm{~s}^{-1}$ (de Zeeuw et al. 1999), as this would have produced a dispersion of $\sigma\left(\mu_{\tau}\right) \simeq 5-6$ mas yr $^{-1}$ in both subgroups.

Table 1. Estimates of $\sigma\left(\mu_{\tau}\right)$ from Monte Carlo Simulations and Observations

\begin{tabular}{lcc} 
Sample & $\begin{array}{c}\mathrm{LCC} \sigma\left(\mu_{\tau}\right) \\
\left(\operatorname{mas~yr}^{-1}\right)\end{array}$ & $\begin{array}{c}\mathrm{UCL} \sigma\left(\mu_{\tau}\right) \\
\left(\text { mas yr }^{-1}\right)\end{array}$ \\
\hline Observed RASS-TYC2 sample & $2.89 \pm 0.46$ & $3.48 \pm 0.49$ \\
\hline Model $\sigma_{\text {int }}^{\star}=0.0 \mathrm{~km} \mathrm{~s}^{-1}$ & 2.62 & 3.18 \\
Model $\sigma_{\text {int }}^{\star}=0.5 \mathrm{~km} \mathrm{~s}^{-1}$ & 2.78 & 3.27 \\
Model $\sigma_{\text {int }}^{\star}=1.0 \mathrm{~km} \mathrm{~s}^{-1}$ & 3.24 & 3.48 \\
Model $\sigma_{\text {int }}^{\star}=1.5 \mathrm{~km} \mathrm{~s}^{-1}$ & 3.84 & 3.89 \\
Model $\sigma_{\text {int }}^{\star}=2.0 \mathrm{~km} \mathrm{~s}^{-1}$ & 4.53 & 4.35 \\
Model $\sigma_{\text {int }}^{\star}=2.5 \mathrm{~km} \mathrm{~s}^{-1}$ & 5.30 & 4.86 \\
Model $\sigma_{\text {int }}^{\star}=3.0 \mathrm{~km} \mathrm{~s}^{-1}$ & 6.08 & 5.41 \\
\hline
\end{tabular}

The velocity dispersions determined from the Monte Carlo simulations are strictly upper limits only. The MML02 survey observed most of the stars in the selection boxes in Fig. 1, and some of those are known not to be pre-MS members. Interlopers will evenly populate the Fig. 1 selection boxes, leading to slightly inflated dispersions in $\mu_{\tau}$, although the use of probability plots largely mitigates against this effect. Taking into account the lack of spectroscopic confirmation of pre-MS status for all of the RASS-TYC2 candidate members, I conservatively conclude the following: The intrinsic velocity dispersion $\sigma_{\text {int }}^{\star}$ of post-T Tauri stars in the $L C C$ and $U C L O B$ subgroups is $\leq 2 \mathrm{~km} \mathrm{~s}^{-1}$, similar to that measured for the early-type members (de Bruijne 1999a). A more detailed study, including radial velocity data, and answering whether the subgroup expansion is detectable, is underway (Mamajek, in prep.).

\section{Is There an OB Subgroup in Chamaeleon?}

Sartori et al. (2003; hereafter SLD03) recently presented a membership list of $21 \mathrm{~B}$ stars in the Chamaeleon region that they claim constitute a new OB subgroup of Sco-Cen ( $\$ 2.3$ and Table 5 of their paper). The putative Cha OB members were selected solely by distance (120- 
$220 \mathrm{pc}$ ) and projected proximity to the Chamaeleon molecular clouds. I present two observations which demonstrate that either the Cha subgroup membership list of SLD03 is severely contaminated by field stars, or that the group doesn't exist.

SLD03 measured a velocity dispersion for their Cha B-star sample of $\left(\sigma_{U}, \sigma_{V}, \sigma_{W}\right)=(8,11,6) \mathrm{km} \mathrm{s}^{-1}$. Observations of nearby OB associations show that their velocity dispersions are small - typically $\leq 1$ $3 \mathrm{~km} \mathrm{~s}^{-1}$ ( $\S 2$; de Zeeuw et al. 1999; de Bruijne 1999). Torra et al. (2000) find that young ( $<100$ Myr-old), nearby $(d=100-600 \mathrm{pc})$ field OB stars distributed all over the sky have a velocity dispersion of $\left(\sigma_{U}, \sigma_{V}, \sigma_{W}\right)$ $\simeq(8,9,5) \mathrm{km} \mathrm{s}^{-1}$. This is similar to the velocity dispersion for the Cha B stars, and suggests that if the sample contains a bona fide OB association, it is probably severely contaminated by field B stars.

There is also a discrepancy in the numbers of Cha OB members versus non-members in the Chamaeleon region. If one searches the Hipparcos catalog for B stars in the $180 \mathrm{deg}^{2}$ region surveyed for Chamaeleon ROSAT T Tauri stars by Alcalá et al. (1995), constrained to distances between $120-220 \mathrm{pc}$, one finds that all $12 \mathrm{~B}$ stars within these constraints are considered Cha OB members by SLD03. Where are the non-member, field B stars? How many would one expect? The projected density of Hipparcos B stars with distances of $120-220$ pc at Galactic latitude $-18^{\circ}$ is $\sim 0.057 \mathrm{deg}^{-2}$ (calculated in a $10^{\circ}$-wide band centered on $b=-18^{\circ}$, covering all Galactic longitudes). Over the $180 \mathrm{deg}^{2}$ Cha region defined by Alcalá, we expect to find $10 \pm \sqrt{10}$ B-type field stars. One finds 12 , consistent with the density of field B-stars, and within the 1- $\sigma$ Poisson error bar. It is difficult to accept that all 12 B-type Hipparcos stars in this region are members of a new $\mathrm{OB}$ association - with zero non-member field B stars - especially when 10 non-member B stars are predicted to exist. These numbers also suggest that there is not a statistically significant over-density of B stars in the Chamaeleon region. Along with the high velocity dispersion of the putative Cha OB membership list, the evidence presented here suggests that there is no OB subgroup in Chamaeleon.

\section{Future Prospects}

Projects are underway to further understand the nature of the LCC and UCL post-T Tauri stars and their circumstellar environs. E. Mamajek, M. Meyer, P. Hinz, \& W. Hoffmann have recently completed a $3-10 \mu \mathrm{m}$ survey for cool accretion disks among $\sim 40$ of the Sco-Cen 
post-T Tauri stars using the MIRAC/BLINC mid-IR imaging system on the Magellan I telescope. It is unclear whether pre-MS stars can retain cooler disks (observed at longer $\lambda$ ) for times longer than the observed lifetime for inner disks traced by JHKL data ( 10 Myr). Further, its unclear whether these disks can regulate stellar angular momentum evolution. Approximately 30 of the LCC and UCL post-T Tauri stars are among the $\sim 350$ young, solar-type stars in the Formation and Evolution of Planetary Systems (FEPS) SIRTF Legacy Project (Meyer et al. 2002). A high resolution echelle survey of the post-T Tauri star sample was conducted in June 2002 on the CTIO 4-m telescope for measuring accurate radial and rotational velocities (Mamajek et al., in prep.). The primary goals are (1) to determine the distribution of stellar rotational velocities and study stellar angular momentum evolution in the post- $\mathrm{T}$ Tauri phase, (2) use radial velocities to ensure membership, as well as determine the kinematic expansion age of the subgroups, and (3) determine whether a spread in $\mathrm{Li}$ abundances is present.

\section{Acknowledgments}

EEM is currently supported by a NASA Graduate Student Researchers Program fellowship (NGT5-50400) and recently by NASA contract 1224768 administered by JPL. I thank the meeting organizers for allowing me to give an oral presentation, and for making the Ouro Preto meeting such an enjoyable success. I also thank Mike Meyer and Lissa Miller for critiquing drafts of this manuscript.

\section{References}

Alcalá, J.M., et al., 1995, A\&A, 114, 109

Barbier-Brossat, M. \& Figon, P., 2000, A\&AS, 142, 217

de Bruijne, J., 1999a, MNRAS, 310, 585

de Bruijne, J., 1999b, MNRAS, 306, 381

Hoogerwerf, R., 2000, MNRAS, 313, 43

Jensen, E. L. N. 2001, ASP Conf. Ser. 244: Young Stars Near Earth: Progress and Prospects, 3

Lutz, T. E., \& Upgren, A. R., 1980, AJ, 85, 1390

Mamajek, E.E., Meyer, M.R., \& Liebert, J., 2002, AJ, 124, 1670 (MML02)

Meyer, M.R., et al., 2002, The Origins of Stars and Planets: The VLT View. Proceedings of the ESO Workshop held in Garching, Germany, 24-27 April 2001, p. 463.

Sartori, M.J., Lépine, J.R.D., \& Dias, W.S., 2003, A\&A, in press (SLD03)

Schmidt-Kaler, Th., 1982, in Landolt-Börnstein: Numerical Data and Functional Relationships in Science and Technology, eds. K. Schaífers \& H. H. Voigt, (Berlin: Springer-Verlag)

Smart, W.M., 1968, Stellar Kinematics, (Longmans Green \& Co Ltd: London)

Torra, J., Fernández, D., \& Figueras, F., 2000, A\&A, 359, 82

de Zeeuw et al., 1999, AJ, 117, 354 